\title{
Epidemiology of Plasmodium falciparum Infections in a Semi-Arid Rural African Setting: Evidence from Reactive Case Detection in Northwestern Kenya
}

\author{
Hannah R. Meredith, ${ }^{1 \star} †$ Amy Wesolowski, ${ }^{1} \dagger$ Diana Menya, ${ }^{2}$ Daniel Esimit, ${ }^{3}$ Gilchrist Lokoel, ${ }^{3}$ Joseph Kipkoech, ${ }^{4}$ Betsy Freedman, ${ }^{5}$ \\ Samuel Lokemer, ${ }^{3}$ James Maragia, ${ }^{6}$ George Ambani, ${ }^{4}$ Steve M. Taylor, ${ }^{5,7}$ Wendy Prudhomme-O’Meara, ${ }^{2,5,7} \ddagger$ and \\ Andrew A. Obala ${ }^{8} \ddagger$ \\ ${ }^{1}$ Department of Epidemiology, Johns Hopkins Bloomberg School of Public Health, Baltimore, Maryland; ${ }^{2}$ Department of Epidemiology and Medical \\ Statistics, School of Public Health, College of Health Sciences, Moi University, Eldoret, Kenya; ${ }^{3}$ Department of Health Services and Sanitation, \\ Turkana County, Kenya; ${ }^{4}$ Academic Model Providing Access to Healthcare, Eldoret, Kenya; ${ }^{5}$ Division of Infectious Diseases, School of Medicine, \\ Duke University, Durham, North Carolina; ${ }^{6}$ Lodwar County Referral Hospital, Turkana County, Kenya; ${ }^{7}$ Duke Global Health Institute, Duke University, \\ Durham, North Carolina; ${ }^{8}$ School of Medicine, College of Health Sciences, Moi University, Eldoret, Kenya
}

\begin{abstract}
In northwestern Kenya, Turkana County has been historically considered unsuitable for stable malaria transmission because of its unfavorable climate and predominantly semi-nomadic population; consequently, it is overlooked during malaria control planning. However, the area is changing, with substantial development, an upsurge in travel associated with resource extraction, and more populated settlements forming. Recently, numerous malaria outbreaks have highlighted the need to characterize malaria transmission and its associated risk factors in the region to inform control strategies. Reactive case detection of confirmed malaria cases at six health facilities across central Turkana was conducted from 2018 to 2019. Infections in household members of index cases were detected by malaria rapid diagnostic tests (RDTs) and PCR tests, and they were grouped according household and individual characteristics. The relationships between putative risk factors and infection were quantified by multilevel logistic regression models. Of the 3,189 household members analyzed, 33.6\% had positive RDT results and/or PCR test results. RDT-detected infections were more prevalent in children; however, PCR-detected infections were similarly prevalent across age groups. Recent travel was rarely reported and not significantly associated with infection. Bed net coverage was low and net crowding was associated with increased risks of household infections. Infections were present year-round, and fluctuations in prevalence were not associated with rainfall. These findings indicate year-round, endemic transmission with moderate population immunity. This is in stark contrast to recent estimates in this area. Therefore, further investigations to design effective intervention approaches to address malaria in this rapidly changing region and other similar settings across the Horn of Africa are warranted.
\end{abstract}

\section{INTRODUCTION}

Large swaths of sub-Saharan Africa are undergoing dramatic demographic transitions that include changing land use and human settlement patterns, particularly in the semiarid and arid areas. These transitions coincide with unprecedented human circulation and decreased isolation of these communities, which are factors known to impact malaria transmission. ${ }^{1}$ It is critical to characterize malaria transmission during these changes to ensure that appropriate control strategies are in place and that malaria does not go unchecked in areas once considered unsuitable for transmission.

One area undergoing such transitions with limited characterization of malaria is Turkana, Kenya. This northwestern Kenyan county has a harsh, semi-arid climate with little rainfall $\left(<215 \mathrm{~mm}\right.$ annually) and temperatures as high as $40^{\circ} \mathrm{C} / 104^{\circ} \mathrm{F}$, thus rendering most of the region unsuitable for agriculture. ${ }^{2,3}$ The region is sparsely populated by nomadic and seminomadic pastoralists; there are fewer than 1.5 million residents across more than $77,000 \mathrm{~km}^{2}{ }^{4,5}$ Rainfall is erratic, with brief, violent storms and high surface runoff possibly creating transient mosquito breeding habitats. ${ }^{6}$ Few studies have characterized the burden of malaria in this region and largely purport limited to no transmission. ${ }^{7,8}$ Consequently, this area has been considered unsuitable for malaria transmission and historically overlooked when planning malaria interventions. ${ }^{9}$

\footnotetext{
*Address correspondence to Hannah Meredith, Johns Hopkins Bloomberg School of Public Health, 615 North Wolfe Street, E6003, Baltimore, MD 21205. E-mail: hmeredi4@jhmi.edu $\dagger$ These authors contributed equally as co-first authors. $\ddagger$ These authors contributed equally as co-senior authors.
}

Recently, this assumption has been challenged by several serious outbreaks. ${ }^{10,11}$ The drivers of these outbreaks are unknown, but they may be attributable to regional development for electric power, oil exploration, and water extraction. ${ }^{12-15}$ The ensuing increase in travel and infrastructure development, establishment of more densely populated settlements, and increase in the presence of surface water may have collectively enhanced the suitability for sustained malaria transmission. To guide malaria control efforts in the region, the malaria burden and its associated risk factor need to be quantified.

We hypothesized that Turkana currently supports low levels of endemic malaria transmission, which have been shown to be associated with higher levels of low-density infections. ${ }^{16}$ These low-density infections are often missed by microscopy or rapid diagnostic tests (RDTs); because the limited studies of malaria in Turkana largely used microscopy, this could be the reason why relatively low levels of malaria have been reported to date. ${ }^{9}$ This was the case in Sudan, where PCR tests revealed that a substantial proportion of submicroscopic cases was present year-round in an area that had been deemed unstable for malaria transmission based on microscopy detection methods. ${ }^{17}$ Low-density infections and Turkana's recent changes creating a more suitable setting for mosquitoes to breed and malaria to spread could be underpinning the increase in recent outbreaks.

Based on the conservative assumption that few cases would be detected in Turkana, we established a reactive case detection of patients with malaria cases presenting to health facilities across central Turkana and their household members. We enrolled both urban and semi-nomadic populations for whom changing demographics, more permanent 
settlements, and land use patterns may affect the likelihood of malaria transmission. ${ }^{18,19}$ Our primary aim was to characterize the infections and risk factors associated with malaria cases in households of confirmed cases. Our secondary objective was to assess the utility of different tests (e.g., RDTs or PCR test) for detecting cases in this setting. Ultimately, these findings will help inform the understanding of malaria transmission and the most effective intervention approaches for this region and other similar settings across the Horn of Africa.

\section{MATERIALS AND METHODS}

Study area. Turkana is a vast county in northwestern Kenya with semi-arid conditions and predominantly semi-nomadic and nomadic populations (Figure 1A). Lodwar is the capital of the county and largest town, with a more urbanized and dense population (50 individuals $/ \mathrm{km}^{2}$ versus 13 individuals/ $\mathrm{km}^{2}$ in the whole county). ${ }^{20}$ We focused on three urban (St. Monica, St. Patrick, and Ngiitakito) and three rural (Nakechichok, Nadoto, and Kerio) health facilities in central Turkana. These facilities are located along a seasonal river (Turkwel) running east that connects Lodwar to Lake Turkana. This transect encompasses a variety of land use (e.g., farming, pastoralism, urban business sites) and water use (e.g., piped water, irrigation canals, rivers) patterns, rates of development, and proportions of families with a (semi-)nomadic lifestyle.

Study population. Between August 2018 and October 2019, we received consent from and enrolled patients at these health facilities with malaria confirmed by the Pf-HRP2 RDT (Carestart). We collected a dried blood spot (DBS) from each patient. Patients also provided responses to a questionnaire detailing their travel and medical history. After enrollment of the first 30 index patients at each facility during each month, a community health worker visited the patient's home within 1 week if they lived within the catchment area of the facility or were visiting for at least 2 weeks. Each consenting household member provided a finger-prick blood sample for an RDT and
DBS and completed a questionnaire. All participants with positive RDT results were prescribed Artemether-Lumefantrine.

Rainfall measurements. A shapefile encompassing the entire study area was used to extract daily rainfall estimates between October 2018 and October 2019 from ClimateSERV (https://climateserv.servirglobal.net/), which were aggregated monthly using $R$ (version 3.6.3). Months with more than $10 \mathrm{~mm}$ of total rain were categorized as rainy (as opposed to dry).

Molecular detection of Plasmodium falciparum. Genomic DNA was extracted from each DBS using Chelex-100, and $P$. falciparum was detected and quantified using a duplex real-time PCR assay targeting the pfr364 motif in parasites as well as human beta-tubulin. ${ }^{21}$ Genomic DNA from each sample was tested in duplicate, and a positive sample was defined as amplification from both replicates or amplification from a single replicate with a cycle threshold $(\mathrm{Ct})$ value $\leq 38$. Densities were estimated from standard curves on each reaction plate computed from a series of standards ranging from 0.1 to 2000 parasites/ $\mu \mathrm{L}$ of whole blood.

Spatial mapping of cases. Each household reported the nearest landmark associated with their location. Using BackCountry Navigator, 127 landmarks were geolocated, aggregated to a $1-\times 1-\mathrm{km}$ grid, and plotted using $\mathrm{R}$. The median density of landmarks within a grid cell was 1 (interquartile range [IQR], 1-14), with the highest density of landmarks being reported in urban catchment areas. In the Kerio catchment area, $129(11.7 \%)$ households whose landmarks could not be collected because of an impassable river were aggregated to a single point across the river. An additional 91 (8.2\%) households were too remote to collect landmarks; therefore, they were mapped using the coordinates of the relevant health facility.

Data capture and statistical analyses. Data from index patients and household members were collected using paper forms and entered into a REDCap database (https://www. project-redcap.org/). Univariate and multivariate logistic regressions were used to analyze individual and household
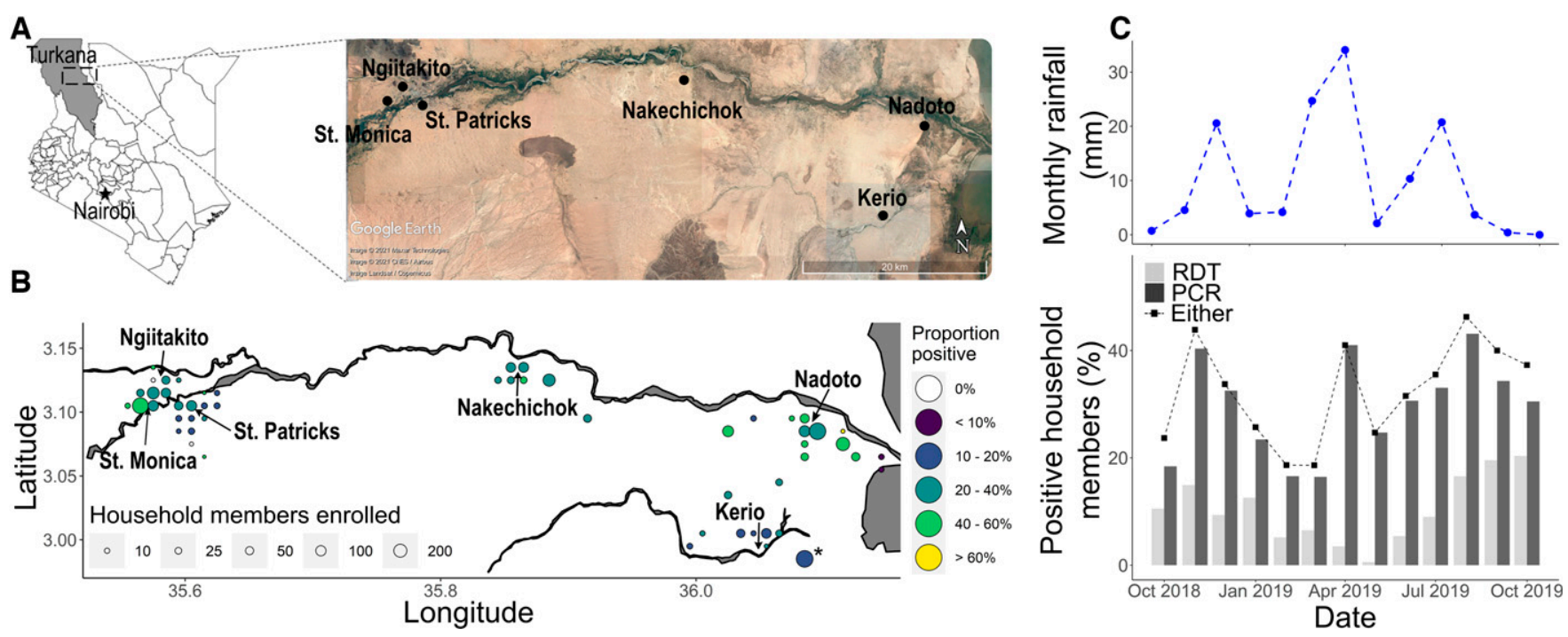

FIGURE 1. The study area, enrollment, and positivity for household members based on the results of rapid diagnostic test (RDTs) and/or PCR tests. (A) The study was performed in central Turkana, which is a county in northwestern Kenya. Index patients from health facilities and their households were enrolled (Map data from Google Earth, Maxar, CNES/Airbus, and Landsat/Copernicus). (B) Spatial distribution of the proportion of household members with positive results for malaria according to RDTs or PCR tests (*household members who were inaccessible for mapping because of seasonal flooding). (C) The percent of household members with positive results for malaria according to RDTs or PCR tests are compared for each month and based on the total monthly rainfall $(\mathrm{mm})$. 
factors related to RDT-detected and PCR-detected infections, with the health facility included as a random effect. Although individuals with RDT-positive/PCR-negative results may represent recently cleared or treated infections rather than current infections, they were included in the analysis because they would have been detected and treated as a positive case by the local health facilities. A householdlevel random effect was not included because of low interclass correlation values, but it did not qualitatively change these results (Supplemental Table S1). Individual variables that were analyzed included demographics (age, sex), net usage, presence of malaria-related symptoms, and sleeping location. Household variables that were analyzed included index case characteristics (age, sex), season enrolled (dry or rainy), any travel (anyone from the household who traveled within the past 2 months), type of water source (open or closed), latrine use, and livestock presence. Householdlevel analyses were also performed using logistic regression for the presence of at least one RDT-detected or PCRdetected infection. Results for households with exclusive RDT-detected or PCR-detected infections are shown in the Supplemental Materials.

\section{RESULTS}

Study population. We enrolled 1933 confirmed malaria cases from six health facilities in central Turkana between August 2018 and October 2019 (Figure 1A, Supplemental Figure 1). Most index patients were 15 years or younger $(59.3 \%$; $1,138 / 1,919)$, and more index patients were enrolled from rural health facilities than from urban ones $(60.2 \% ; 1,164 / 1,933)$ (Supplemental Table 2). Nearly all index cases were found in residents rather than in visitors $(93.5 \% ; 1,807 / 1,932)$, and few reported recent travel $(5.4 \% ; 103 / 1,923)$. Among those who reported travel, the majority traveled within Turkana County (Supplemental Table 3).

A household investigation of 1,104 index patients resulted in the enrollment of 3,353 household members. We obtained the molecular results of all enrolled members of 1,042 (94.4\%) households comprising 3,189 household members. The median reported household size was 4 (IQR, 3-6), and the median number of household members enrolled was 3 (IQR, $2-4)$. Most household members enrolled were 15 years or younger $(48.6 \% ; 1,538 / 3,167)$, from a rural catchment area (62.7\%; 1,998/3,189), and reported no symptoms (84.6\%; $2,697 / 3,189)$ (Table 1). The majority of participants from rural areas slept outside (89.5\%; 1,759/1,966), whereas the majority of those from urban areas slept inside $(59.0 \%$; 682/1,155). Few individuals reported using a bed net $(20.3 \%$; 634/3,119), and only $6.3 \%$ of households (60/951) reported having enough bed nets to meet the WHO recommended distribution density of one net for every two people (Tables 1 and 2). ${ }^{22}$ Overnight travel was rare; 1 out of every 10 households had at least one member (including the index patient) who recently traveled, and only $1.9 \%(60 / 3,180)$ of household members (not including the index patient) reported travel. The majority of trips remained within Turkana County (Supplemental Table 3).

Malaria prevalence and distribution. Excluding index patients, the $P$. falciparum prevalence among household members was $11.4 \%(365 / 3,189)$ according to the RDT,

TABLE 1

Characteristics of all index patients and household members as well as those with positive RDT and/or PCR test results for malaria

\begin{tabular}{|c|c|c|c|c|c|}
\hline & \multirow{2}{*}{$\begin{array}{c}\text { Index patient, \% (number/N) } \\
\text { All } \\
(N=1,042) \\
\end{array}$} & \multicolumn{4}{|c|}{ Household members, \% (number/N) } \\
\hline & & $\begin{array}{c}\text { All } \\
(N=3,189)\end{array}$ & $\begin{array}{l}\text { Positive results according to } \\
\text { any test ( } \mathrm{N}=\# \text { in category) }\end{array}$ & $\begin{array}{l}\text { Positive RDT results } \\
(\mathrm{N}=\# \text { in category })\end{array}$ & $\begin{array}{c}\text { Positive PCR test results } \\
(\mathrm{N}=\# \text { in category })\end{array}$ \\
\hline \multicolumn{6}{|l|}{$\overline{\text { Age }}$} \\
\hline 15 years or younger & $57.1(594 / 1,041)$ & $48.6(1,538 / 3,167)$ & $36.5(561 / 1,538)$ & $15.8(243 / 1,538)$ & $32.6(501 / 1,538)$ \\
\hline $16-40$ years & $34.9(363 / 1,041)$ & $41.4(1,312 / 3,167)$ & $30.6(401 / 1,312)$ & $7.6(100 / 1,312)$ & $28.7(377 / 1,312)$ \\
\hline Older than 40 years & $8.1(84 / 1,041)$ & $10.0(3,17 / 3,167)$ & $31.6(100 / 317)$ & $6.9(22 / 317)$ & $29.0(92 / 317)$ \\
\hline \multicolumn{6}{|l|}{$\begin{array}{l}\text { Health facility of index } \\
\text { patient }\end{array}$} \\
\hline Rural & $63.7(664 / 1,042)$ & $62.7(1,998 / 3,189)$ & $32.9(657 / 1,998)$ & $8.9(178 / 1,998)$ & $31.0(620 / 1,998)$ \\
\hline Kerio & $19.6(204 / 1,042)$ & $19.3(614 / 3,189)$ & $19.2(118 / 614)$ & $0.2(1 / 614)$ & $19.1(117 / 614)$ \\
\hline Nadoto & $29.8(311 /, 1042)$ & $29.1(928 / 3,189)$ & $41.6(386 / 928)$ & $14.1(131 / 928)$ & $38.7(359 / 928)$ \\
\hline Nakechichok & $14.3(149 / 1,042)$ & $14.3(456 / 3,189)$ & $33.6(153 / 456)$ & $10.1(46 / 456)$ & $31.6(144 / 456)$ \\
\hline Urban & $36.3(378 / 1,042)$ & $37.3(1,191 / 3,189)$ & $34.7(413 / 1,191)$ & $15.7(187 / 1,191)$ & $30.1(358 / 1,191)$ \\
\hline Ngiitakito & $12.7(132 / 1,042)$ & $13.3(425 / 3,189)$ & $35.1(149 / 425)$ & $17.4(74 / 425)$ & 29.7 (126/425) \\
\hline St. Monica & $12.1(126 / 1,042)$ & $14.4(458 / 3,189)$ & $43.7(200 / 458)$ & $20.5(94 / 458)$ & $39.1(179 / 458)$ \\
\hline \multirow[t]{2}{*}{ St. Patrick } & $11.5(120 / 1,042)$ & $9.7(308 / 3,189)$ & $20.8(64 / 308)$ & $6.2(19 / 308)$ & $17.2(53 / 308)$ \\
\hline & $\begin{array}{c}\text { All } \\
(N=1,042)\end{array}$ & $\begin{array}{c}\text { All } \\
(N=3,189)\end{array}$ & $\begin{array}{l}\text { Positive results according } \\
\text { to any test }(N=1,070)\end{array}$ & $\begin{array}{l}\text { Positive RDT results } \\
\qquad(N=365)\end{array}$ & $\begin{array}{l}\text { Positive PCR results } \\
\qquad(N=978)\end{array}$ \\
\hline$\overline{\text { Male }}$ & $46.8(488 / 1,042)$ & $43.4(1,380 / 3,183)$ & $44.3(474 / 1,069)$ & $51.5(188 / 365)$ & $43.4(424 / 977)$ \\
\hline Personal travel* & $4.7(48 / 1,032)$ & $1.9(60 / 3,180)$ & $1.6(17 / 1,068)$ & $1.9(7 / 365)$ & $1.4(14 / 976)$ \\
\hline \multicolumn{6}{|l|}{ Symptoms } \\
\hline Any & $99.3(1026 / 1,033)$ & $15.4(492 / 3,189)$ & $28.2(302 / 1,070)$ & $67.4(246 / 365)$ & $24.5(240 / 978)$ \\
\hline Fever & $83.8(866 / 1,033)$ & $6.2(198 / 3,189)$ & $15.4(165 / 1,070)$ & $41.6(152 / 365)$ & $12.7(124 / 978)$ \\
\hline Headache & $84.6(874 / 1,033)$ & $6.9(219 / 3,189)$ & $16.2(173 / 1,070)$ & $44.1(161 / 365)$ & $13.8(135 / 978)$ \\
\hline Body aches & $31.8(329 / 1,033)$ & $1.8(56 / 3,189)$ & $3.5(37 / 1,070)$ & $8.2(30 / 365)$ & $3.27(32 / 978)$ \\
\hline None & $0.7(7 / 1,033)$ & $84.6(2,697 / 3,189)$ & $71.8(768 / 1,070)$ & $32.6(119 / 365)$ & $75.46(738 / 978)$ \\
\hline \multicolumn{6}{|l|}{$\begin{array}{l}\text { Sleeping details of the } \\
\text { previous night }\end{array}$} \\
\hline Used bed net & $20.7(216 / 1,041)$ & $20.3(634 / 3,119)$ & $21.3(223 / 1,048)$ & $25.4(90 / 355)$ & $20.79(199 / 957)$ \\
\hline Slept outside & $69.7(723 / 1,037)$ & $71.5(2,232 / 3,121)$ & $73.6(770 / 1,046)$ & $67.4(240 / 356)$ & $74.97(716 / 955)$ \\
\hline
\end{tabular}

$\mathrm{RDT}=$ rapid diagnostic test. Missing data resulted in the denominators varying for some characteristics.

* Personal travel refers to the household member reporting any travel. 
TABLE 2

Characteristics of all households as well as those with at least one additional infection

\begin{tabular}{|c|c|c|c|c|}
\hline & $\begin{array}{c}\text { All } \\
(N=1,042)\end{array}$ & $\begin{array}{l}\geq 1 \text { Positive result according } \\
\text { to any test ( } \mathrm{N}=\# \text { in category) }\end{array}$ & $\begin{array}{l}\geq 1 \text { Positive RDT result } \\
(\mathrm{N}=\# \text { in category })\end{array}$ & $\begin{array}{l}\geq 1 \text { Positive PCR result } \\
(\mathrm{N}=\# \text { in category })\end{array}$ \\
\hline \multicolumn{5}{|l|}{ Health facility } \\
\hline Rural & $62.9(638 / 1,014)$ & $57.4(381 / 664)$ & $17.9(119 / 664)$ & $56(372 / 664)$ \\
\hline Kerio & $18.8(191 / 1,014)$ & $41.2(84 / 204)$ & $0.5(1 / 204)$ & $40.7(83 / 204)$ \\
\hline Nadoto & $29.6(300 / 1,014)$ & $66.9(208 / 311)$ & $28.6(89 / 311)$ & $65(202 / 311)$ \\
\hline Nakechichok & $14.5(147 / 1,014)$ & $59.7(89 / 149)$ & $19.5(29 / 149)$ & $58.4(87 / 149)$ \\
\hline Urban & $37.1(376 / 1,014)$ & $58.5(221 / 378)$ & $31(117 / 378)$ & $54(204 / 378)$ \\
\hline Ngiitakito & $12.9(131 / 1,014)$ & $61.4(81 / 132)$ & $38.6(51 / 132)$ & $56.1(74 / 132)$ \\
\hline St. Monica & $12.4(126 / 1,014)$ & $74.8(95 / 127)$ & $39.4(50 / 127)$ & $70.9(90 / 127)$ \\
\hline \multirow[t]{2}{*}{ St. Patrick } & $11.7(119 / 1,014)$ & $37.8(45 / 119)$ & $13.4(16 / 119)$ & $33.6(40 / 119)$ \\
\hline & $\begin{array}{c}\text { All } \\
(N=1,042)\end{array}$ & $\begin{array}{c}\geq 1 \text { Positive result according } \\
\text { to any test }(N=592)\end{array}$ & $\begin{array}{c}\geq 1 \text { Positive RDT result } \\
(N=236)\end{array}$ & $\begin{array}{c}\geq 1 \text { Positive PCR result } \\
(N=576)\end{array}$ \\
\hline \multicolumn{5}{|l|}{ Household details } \\
\hline Buildings & $3(2-3)$ & $3(2-3)$ & $3(2-3)$ & $3(2-3)$ \\
\hline Any travelers & $9.5(95 / 1,004)$ & $7.7(46 / 597)$ & $6.8(16 / 234)$ & $7.7(44 / 571)$ \\
\hline Latrine & $27.9(282 / 1,012)$ & $24.1(145 / 601)$ & $28.0(66 / 236)$ & $23.0(132 / 575)$ \\
\hline Livestock at the homestead & $69.6(704 / 1,012)$ & $73.2(440 / 601)$ & $72.0(170 / 236)$ & $73.9(425 / 575)$ \\
\hline Members (n) & $4(3-6)$ & $5(4-6)$ & $5(4-7)$ & $5(4-6)$ \\
\hline \multicolumn{5}{|l|}{ Children (n) } \\
\hline 0 & $26.5(264 / 996)$ & $18.9(112 / 592)$ & $10.4(24 / 231)$ & $18.7(106 / 566)$ \\
\hline $1-2$ & $55.4(552 / 996)$ & $54.2(321 / 592)$ & $52.4(121 / 231)$ & $53.9(305 / 566)$ \\
\hline$\geq 3$ & $18.1(180 / 996)$ & $26.9(159 / 592)$ & $37.2(86 / 231)$ & $27.4(155 / 566)$ \\
\hline \multicolumn{5}{|l|}{ Bed nets in household } \\
\hline $1-2$ individuals/net & $6.3(60 / 951)$ & $4.6(26 / 562)$ & $3.7(8 / 218)$ & $4.5(24 / 537)$ \\
\hline $2-4$ individuals/net & $10.5(100 / 951)$ & $11(62 / 562)$ & $13.3(29 / 218)$ & $10.6(57 / 537)$ \\
\hline$>4$ individuals $/$ net & $13.1(125 / 951)$ & $16.7(94 / 562)$ & $24.3(53 / 218)$ & $16.8(90 / 537)$ \\
\hline 0 nets & $70(666 / 951)$ & $67.6(380 / 562)$ & $58.7(128 / 218)$ & $68.2(366 / 537)$ \\
\hline Any sleeping outside & $76.8(779 / 1,014)$ & $80.6(485 / 602)$ & $75(177 / 236)$ & $81.2(468 / 576)$ \\
\hline \multicolumn{5}{|l|}{ Water source } \\
\hline Open water source* & $63.5(644 / 1,014)$ & $64.5(388 / 602)$ & $63.1(149 / 236)$ & $65.6(378 / 576)$ \\
\hline Closed water source $†$ & $38.7(392 / 1,014)$ & $38.4(231 / 602)$ & $42.4(100 / 236)$ & $37.3(215 / 576)$ \\
\hline \multicolumn{5}{|l|}{ Source of income } \\
\hline Formally used & $4.3(44 / 1,014)$ & $3.5(21 / 602)$ & $4.7(11 / 236)$ & $3.1(18 / 576)$ \\
\hline Informally used $\ddagger$ & $38.4(389 / 1,014)$ & $36.2(218 / 602)$ & $39.4(93 / 236)$ & $35.8(206 / 576)$ \\
\hline Agrarian§ & $57.2(580 / 1,014)$ & $60.6(365 / 602)$ & $55.9(132 / 236)$ & $61.5(354 / 576)$ \\
\hline Season enrolled (rainy) & $40.0(417 / 1,042)$ & $61.6(371 / 602)$ & $74.6(176 / 236)$ & $60.6(349 / 576)$ \\
\hline
\end{tabular}

$30.7 \%(978 / 3,189)$ according to the PCR test, and $33.6 \%$ $(1,070 / 3,189)$ according to either the RDT or the PCR test (Table 1). The prevalence of infections was highest among household members enrolled at the urban site of St. Monica $(43.7 \%$; 200/458), and it was lowest at the rural site of Kerio (19.2\%; 118/614) (Figure 1A, Table 1). Generally, RDTs detected fewer cases in rural areas $(27.1 \%$; 178/657) than in urban areas (45.3\%; 187/413). Household member infections varied within catchment areas (Figure 1B), with Nadoto and St. Monica having a geographic prevalence between $40 \%$ and $60 \%$. Although the prevalence detected by RDTs was twice as high for younger age groups $(15.8 \%$ for those younger than 15 years; otherwise, 6.9-7.6\%), the prevalence detected using PCR tests was similar across age groups ( $\approx 28.7-32.6 \%$ ) (Table 1). The prevalence of infections fluctuated over time, with the monthly proportion ranging from 0.6 $\%$ to $20.3 \%$ for RDTs and $16.5 \%$ to $43.1 \%$ for PCR tests (Figure 1C). The prevalence of PCR-detected infections reached a peak of $41 \%$ for household members in April and RDT-detected cases decreased to their nadir of $<1 \%$. The timing of the peaks was not predicted by rainfall patterns.

The majority of household members $(67.4 \%$; $246 / 365)$ with positive RDT results for malaria reported symptoms, with fever and headache being the most common. However, only $24.5 \%$ (240/978) of patients with positive PCR results reported symptoms. Infections detected by PCR alone had a significantly lower mean parasite density $(95.2 \pm 1,275.5 \mathrm{p} / \mu \mathrm{L})$ than those detected by RDTs for household members $(690.7 \pm 3,142.4$ $\mathrm{p} / \mu \mathrm{L} ; P<0.0001)$ and index cases $(3,662.6 \pm 9,091.7 \mathrm{p} / \mu \mathrm{L}$; $P<0.0001$ ) (Figure 2A). Infections detected only by PCR occurred across age categories and sites, ranging from $63.3 \%$ $(316 / 499)$ to $84.8 \%(78 / 92)$ of all infections in children (15 years or younger) and adults (older than 40 years), respectively, and $77.3 \%$ (479/620) compared with 62.9\% (224/356) of all infections in rural and urban sites, respectively (Figure $2 \mathrm{~B}$ and $\mathrm{C}$ ).

Risk factors for malaria. Fully adjusted individual-level models identified characteristics associated with infection and allowed us to compare risk factors for all infections as well as those detected by RDTs or PCR tests (Table 3). The odds of a household member testing positive for malaria by either method were substantially lower if a participant had access to a latrine (adjusted odds ratio [aOR], 0.79; $95 \% \mathrm{Cl}$, 0.62-1.00) or was in a household where at least one member reported recent travel (aOR, 0.64; 95\% Cl, 0.42-0.97). The odds of testing positive increased among those who reported any symptoms (aOR, 12.96; 95\% Cl, 8.23-20.39), reported 

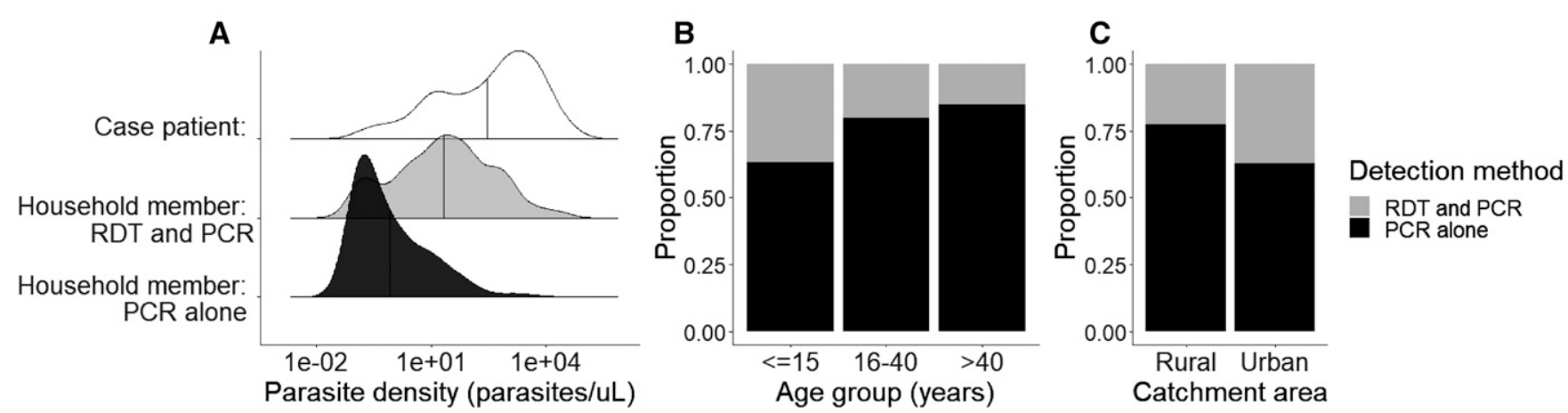

FIGURE 2. Plasmodium falciparum infections in index patients and household members. (A) Parasite density distributions of index patients and household members with infections detectable by rapid diagnostic tests (RDTs) or PCR tests alone. Vertical line indicates the mean parasite density for each group. The proportion of household members was stratified by different test outcomes for age (B) and urban and rural catchment areas (C).

sleeping outdoors (aOR, 1.35; 95\% Cl, 1.09-1.69), and reported using a bed net (aOR, 1.26; 95\% Cl, 1.00-1.58). Age, sex, water source, and exposure to livestock were not significantly associated with the odds of testing positive in general or according to PCR tests; however, younger ages and open water source were significantly associated with increased odds of testing positive according to RDTs. Although symptoms were significantly associated with the odds of testing positive according to RDTs and PCR tests, the strength of association was an order of magnitude greater for RDT-detected cases.

The majority of households $(56.8 \% ; 592 / 1,042)$ had at least one additional case, with $22.6 \%(236 / 1,042)$ having at least one RDT-detected infection and 55.3\% $(576 / 1,042)$ having at least one PCR-detected infection (Table 2). Because of the time and resources necessary for reactive case detection, it would be useful to determine which households have a high probability of additional infections without having to visit the household. Therefore, we developed a model to determine the odds of finding at least one additional infection in a household using individual and household characteristics that could be gathered from index cases presenting at health facilities. Regardless of the detection method, the odds of a household having at least one more infection was associated with the number of household members (aOR, 1.12; 95\% Cl, 1.02-1.24), particularly children, and the presence of at least one person reporting symptoms (aOR, 7.39; 95\% Cl, 3.42-15.99) (Table 4). This is consistent with the individuallevel model, which predicts that symptomatic individuals are more likely to be infected and children are more likely to have an RDT-detected infection. If a household only had infections detectable by PCR testing alone, then symptoms were not associated with the odds of detecting another infection (Supplemental Table 4). Months with more rain (>10 mm) were not associated with the odds of detecting another case using PCR tests; however, they were associated with detecting another case using RDTs (Table 4). Characteristics of the index case (age or sex), reports of any household members traveling, water source, presence of livestock, or the availability of a latrine were not good predictors of detecting another case using either test.

The household-level analysis shed further light on the unexpected result of an increased risk of infection among users of bed nets. When stratifying net ownership by the ratio of nets to household members, net ownership was not significantly associated with an increased odds of additional cases when the net to member ratio is $1: 2$ compared to not owning a net (aOR, 1.04; 95\% Cl, 0.58-1.87) (Table 4). However, when a household reported having a lower net ratio, whereby more than two people would need to share a net, the odds of having additional infections in the household increased compared to that of households with no nets (2-4 individuals per net: aOR, 1.74; 95\% Cl, 1.02-2.95; > 4 individuals per net: aOR, 2.30; $95 \% \mathrm{Cl}, 1.19-4.47)$.

\section{DISCUSSION}

Our reactive case detections of more than 1,000 passively detected malaria cases in Turkana, Kenya, revealed much higher than anticipated infection rates among household members; the prevalence rates were $11.4 \%$ according to RDTs and $30.7 \%$ according to PCR tests. Most RDTdetected cases were found in children, but PCR-detected infections were equally prevalent across age groups. Overnight travel was rarely reported, and most travelers stayed within the county. These findings are indicative of local transmission rather than continuous importation events resulting in local outbreaks. Furthermore, the large number of PCRdetected infections across all age groups is more consistent with stable, rather than emerging, transmission.

The prevalence rate of infection among household members according to PCR tests exceeded $30 \%$, and $56.8 \%$ of households harbored at least one additional infection. Compared with analogous observations from elsewhere in Kenya, PCR testing indicated that the prevalence among household members of index cases was less than $78 \%$, which was observed in a holoendemic setting, ${ }^{21}$ but more than $29 \%$, which was observed in a mesoendemic setting. ${ }^{23}$ In contrast, the prevalence among household members screened during reactive case detection in pre-elimination settings was $<1 \%$ in Cambodia using PCR tests ${ }^{24}$ and $2 \%$ in Eswatini using RDTs. ${ }^{25}$ Because of the vulnerability of Turkana County to significant outbreaks, ${ }^{10}$ and because of the likelihood that this will be exacerbated by land use changes with ongoing development, future population-based studies should describe the general prevalence of $P$. falciparum across the county.

Our results highlight the difficulty sustaining high intervention coverage in this population. The low rate of net use likely reflects challenges with distributing nets to these remote and sometimes mobile households, along with the difficulty of 


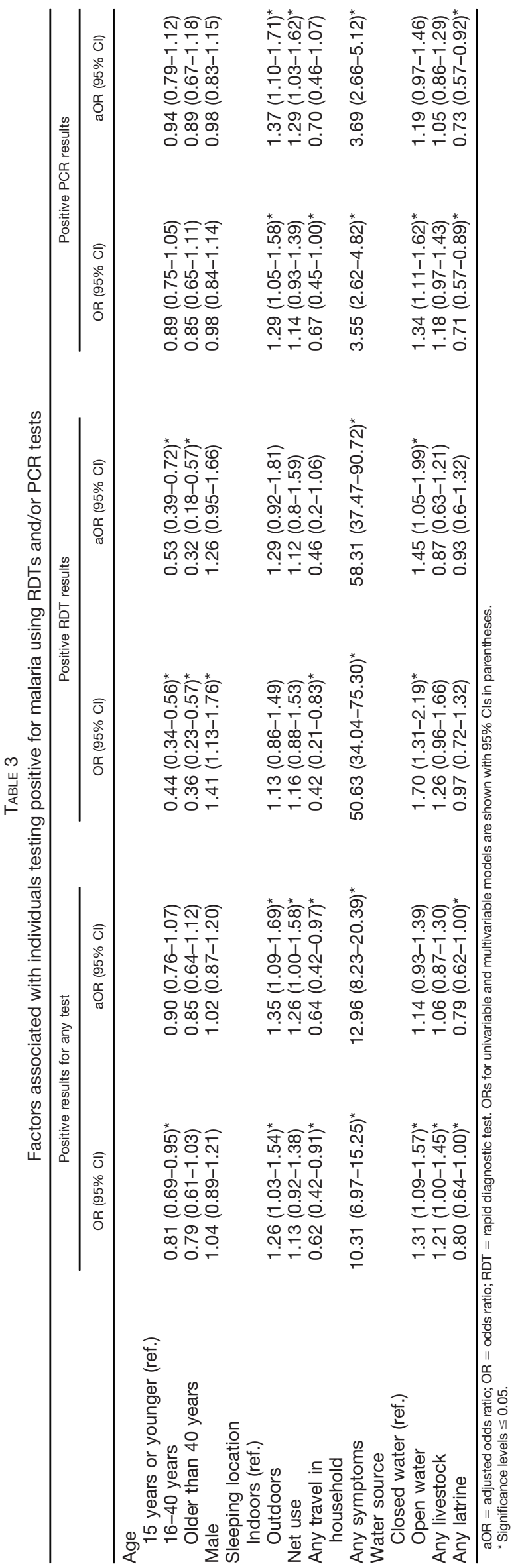

erecting a net over an outdoor sleeping space. Sleeping outdoors was common and accompanied by an increased risk of infection. With such low net coverage, nets would be unlikely to provide any indirect protection to non-net users. ${ }^{26}$ Our results also highlight how bed net quantity and crowding in households undermine bed net efficacy. Compared with households with no nets, those with one net for three or more individuals had higher likelihoods of infections. This underscores the need to capture metrics of bed net distribution and use beyond simple coverage.

Index case enrollment and household member infection prevalence were geographically and temporally heterogenous. Although trends in putative risk factors, such as net use, travel, water source, and sleeping outside, were divided clearly between urban and rural areas, trends in infection were not consistent with urban and rural differences or with a gradient of transmission from the regional capital toward the lake (in the direction of increasing isolation from human circulation); furthermore, they were not synchronized with rainfall. The highest numbers of infections were found in one rural (Nadoto) and one urban (St. Monica) catchment, and the lowest household infection proportions were, again, found in one rural and one urban catchment. The community around Nadoto (high prevalence) was the only area that reported farming as an important livelihood activity. Farming relies on informal irrigation schemes where vectors may breed in stagnant backwaters or residual pools. Local variations in risk factors, possibly including those related to the seasonal Turkwel River, community irrigation schemes, or collecting and using water, need to be further explored to inform locally relevant prevention and environmental management strategies. ${ }^{27}$

Because reactive case detection would be operationally challenging to sustain in a high-transmission setting, and because low levels of malaria interventions (no indoorresidual spraying, low bed net coverage) are implemented in this area, we recast our results to inform possible interventions that could be deployed in this population, particularly community-based approaches. With high numbers of infected individuals throughout the year, seasonal malaria chemoprevention may not be applicable in this setting, despite it being an arid area similar to the Sahel. ${ }^{28}$ Furthermore, the very high levels of subpatent infections indicate that mass testing and treating based on conventional diagnostics may not be effective. Distributing malaria treatment only to RDT-positive individuals would miss a substantial number of infections. Mass drug administration on its own, although shown to be effective in some instances, is less likely to be adopted in this area because of the low levels of other interventions present. ${ }^{29}$ Index case characteristics were not reliable predictors of which households to test and treat in this area; this was in contrast to the findings from another study focused on a low-transmission setting in Eswatini. ${ }^{30}$ This could be because of situational differences in transmission rates $(<1 \%$ versus $>30 \%$ in Eswatini and Turkana, respectively), vector control coverage (40\% versus $20 \%$ ), and occupational risks (farming in a temperate climate versus farming in a semi-arid climate). Instead of relying on index case characteristics, an effective strategy for this area may be household reactive focal drug administration targeting households with more than three children, bed net crowding, and members sleeping outside. Reactive focal drug administration has been shown to be more effective than reactive case detection in a low- 
MEREDITH, WESOLOWSKI, AND OTHERS

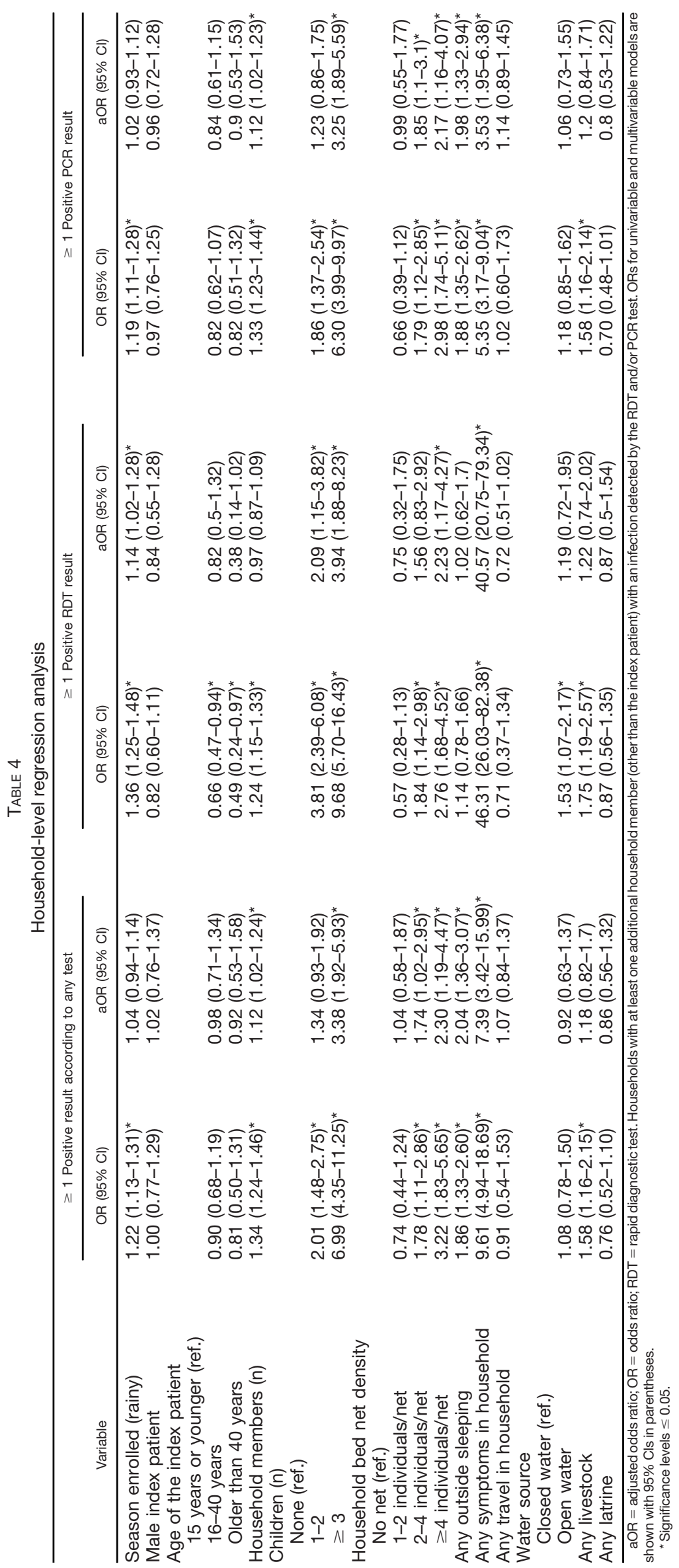


transmission area, ${ }^{31}$ but these findings may not be generalizable to all settings. ${ }^{32} \mathrm{~A}$ better understanding of the infections in households without an index case in the study area would be required to fully evaluate the suitability of this approach; however, previous studies suggest that clustering of cases in an index case household will be significant. ${ }^{33}$

The limitations of this study include a lack of information regarding a population-based sample including households without index cases. This prevented us from understanding the extent of infections in the community. However, it seems likely that infections are fairly widespread considering the preponderance of low-density asymptomatic infections reported here, and that they could be verified by future studies implementing a population-representative cross-sectional survey. Most of the variables we explored were based on self-report; therefore, we cannot rule out under-reporting (of net or livestock ownership), over-reporting (of symptoms), and recall bias. In an effort to reduce recall bias, travel and medical history questions were limited to the past 2 months, which may have excluded more distal, but relevant, information. Finally, this study detected only $P$. falciparum infections; however, the presence and importance of nonfalciparum species need to be explored further because of the contiguous border with Ethiopia and Sudan, which have substantial $P$. vivax transmission rates. ${ }^{34,35}$

\section{CONCLUSIONS}

Our findings suggest that, contrary to expectations, $P$. falciparum is endemic in Turkana. This demonstrates how little is known about malaria transmission in communities such as these that stretch across the Horn of Africa. The high proportion of low-density infections highlights the need for detection methods with higher sensitivity than the standard RDTs as well as treatment and intervention strategies that are compatible with the lifestyle of the community. How long malaria has been circulating locally in this extremely sparsely populated and arid environment is unknown. More research is required to elucidate the relationships among development, demographic changes, and disease risk in these potentially vulnerable populations.

Received March 2, 2021. Accepted for publication May 24, 2021.

Published online August 2, 2021.

Note: Supplemental materials, tables, and figures appear at www. ajtmh.org.

Acknowledgments: We thank our field team, especially Dennis Okoth, Samuel Karanja, Elvis Ekitela, Jackson Kapelo, Rose Adome, Valentine Ramati, Naomi Eyanai, Sister Florence Wafula, and Ruth Areman, and all the families who gave their valuable time to participate in this study. We also thank Kelsey Sumner for her help with preparing the molecular data files.

Disclosure: Written informed consent was obtained from all participants. The study procedures were approved by the ethics review board of Moi University and Duke University. The datasets used and/ or analyzed during the current study are available from the corresponding author on reasonable request.

Financial support: This work was supported by the National Institutes of Allergy and Infectious Diseases of the National Institutes of Health (R21Al133013 to W. P. O.). A. W. is funded by a Career Award at the Scientific Interface by the Burroughs Wellcome Fund, and by the National Library of Medicine of the National Institutes of Health (DP2LM013102). The content is solely the responsibility of the authors and does not necessarily represent the official views of the National Institutes of Health.

Authors' addresses: Hannah R. Meredith and Amy Wesolowski, Department of Epidemiology, Johns Hopkins Bloomberg School of Public Health, Baltimore, MD, E-mails: hmeredi4@jhmi.edu and awesolowski@jhu.edu. Diana Menya, Department of Epidemiology and Medical Statistics, School of Public Health, College of Health Sciences, Moi University, Eldoret, Kenya, E-mail: dianamenya@ gmail.com. Daniel Esimit, Gilchrist Lokoel, and Samuel Lokemer, Department of Health Services and Sanitation, Turkana County, Kenya, E-mails: esimitdaniel@gmail.com, lockhell80@gmail.com, and lokemer0999@gmail.com. Joseph Kipkoech and George Ambani, Academic Model Providing Access to Healthcare, Eldoret, Kenya, E-mails: josepheddykipkoech@gmail.com and georgeambani@gmail.com. Betsy Freedman, Steve M. Taylor, and Wendy Prudhomme-O'Meara, Division of Infectious Diseases, School of Medicine, Duke University, Durham, NC, E-mails: betsy.freedman@ duke.edu, steve.taylor@duke.edu, and wendy.omeara@duke.edu. James Maragia, Lodwar County Referral Hospital, Turkana County, Kenya, E-mail: mjmaragia@gmail.com. Andrew A. Obala, School of Medicine, College of Health Sciences, Moi University, Eldoret, Kenya, E-mail: andrew.obala@gmail.com.

\section{REFERENCES}

1. Wesolowski A, Eagle N, Tatem AJ, Smith DL, Noor AM, Snow RW, Buckee CO, 2012. Quantifying the impact of human mobility on malaria. Science 338: 267-270.

2. National Centers for Environmental Information (NCEI), 2019. Climate Data Online (CDO). Available at: https://www.ncdc.noaa. gov/cdo-web/. Accessed February 17, 2019.

3. United Nations, 2016. Turkana County \& UN Joint Integrated Areabased Development Programme. Available at: www.ke.one.un. org. Accessed November 28, 2020.

4. WorldPop, 2019. WorldPop. Available at: https://www.worldpop. org/. Accessed February 17, 2019.

5. Kenya Food Security Steering Group, Turkana County Steering Group, 2018. Turkana County 2018 Long Rains Food Security Assessment Report Vol. 1. Available at: http://www.ndma.go. ke/index.php/resource-center/send/59-2018/4966-turkanacounty-Ira-2018-report-final. Accessed February 5, 2019.

6. Little MA, Dyson-Hudson R, McCabe JT, 1999. Turkana Herders of the Dry Savanna: Ecology and Biobehavioral Response of Nomads to an Uncertain Environment. Little MA, Leslie PW, eds. Oxford, UK: Oxford University Press.

7. Snow RW, Okiro EA, Noor AM, Munguti K, Tetteh G, Juma E, 2009. The coverage and impact of malaria intervention in Kenya 2007-2009. Division of Malaria Control, Ministry of Public Health and Sanitation.

8. Malaria Atlas Project, 2021. The Malaria Atlas Project. Available at: https://malariaatlas.org/. Accessed February 25, 2021.

9. Ministry of Health, 2016. The epidemiology and control profile of malaria in Kenya: reviewing the evidence to guide the future vector control. National Malaria Control Programme, Ministry of Health. Technical support provided by the LINK Project (London School of Hygiene and Tropical Medicine and the Information for Malaria (INFORM) Project, KEMRI-Wellcome Trust Research Programme), Nairobi, Kenya.

10. MSF, 2019. MSF Responds to Malaria Outbreak in Turkana, Kenya. Available at: https://msf.or.ke/en/magazine/msfresponds-malaria-outbreak-turkana-kenya. Accessed October 16, 2019.

11. Mulambalah C, 2018. An evolving malaria epidemic in Kenya: a regional alert. CHRISMED J Heal Res 5: 162.

12. Agade KM, 2014. Ungoverned space' and the oil find in Turkana. Round Table 103: 497-515.

13. Snyder DE, Wiseman S, Cruthers LR, Slone RL, 2011. Ivermectin and milbemycin oxime in experimental adult heartworm (Dirofilaria immitis) infection of dogs. J Vet Intern Med 25: 61-64.

14. Avery S, 2012. Lake Turkana and the Lower Omo: Hydrological Impacts of Major Dam and Irrigation Developments. Available at: $\quad$ https://www.africanstudies.ox.ac.uk/sites/default/files/ 
africanstudies/documents/media/executive_summary introduction.pdf. Accessed November 28, 2020.

15. UNESCO, 2013. Strategic Groundwater Reserves Found in Northern Kenya. UNESCOPRESS. Available at: http://www.unesco. org/new/en/media-services/single-view/news/strategic_ groundwater_reserves_found_in_northern_kenya/ \#.VYGqtfmqqko. Accessed December 19, 2020.

16. Okell LC, Ghani AC, Lyons E, Drakeley CJ, 2009. Submicroscopic Infection in Plasmodium falciparum-endemic populations: a systematic review and meta-analysis. 1509, doi: 10.1086/ 644781.

17. Roper C, Elhassan IM, Hviid L, Giha H, Richardson W, Babiker H, Satti GMH, Theander TG, Arnot DE, 1996. Detection of very low level Plasmodium falciparum infections using the nested polymerase chain reaction and a reassessment of the epidemiology of unstable malaria in Sudan. Am J Trop Med Hyg 54: 325-331.

18. Baeza A, Bouma MJ, Dhiman RC, Baskerville EB, Ceccato P, Yadav RS, Pascual M, 2013. Long-lasting transition toward sustainable elimination of desert malaria under irrigation development. Proc Natl Acad Sci USA 110: 15157-15162.

19. Garg A, Dhiman RC, Bhattacharya S, Shukla PR, 2009. Development, malaria and adaptation to climate change: a case study from India. Environ Manage 43: 779-789.

20. Kenya National Bureau of Statistics, 2019. Distribution of Population by Administrative Units: 2019 Kenya Population and Housing Census Vol 2. Available at: http://www.knbs.or.ke.

21. Taylor SM, Sumner KM, Freedman B, Mangeni JN, Obala AA, O'Meara WP, 2019. Direct estimation of sensitivity of Plasmodium falciparum rapid diagnostic test for active case detection in a high-transmission community setting. Am J Trop Med Hyg 101: 1416-1423.

22. World Health Organization, 2017. Achieving and Maintaining Universal Coverage with Long-lasting Insecticidal Nets for Malaria Control. (No. WHO/HTM/GMP/2017.20). World Health Organization

23. Aidoo EK et al., 2018. Reactive case detection of Plasmodium falciparum in western Kenya highlands: effective in identifying additional cases, yet limited effect on transmission. Malar $J$ 17: 111.

24. Hustedt $\mathrm{J}$ et al., 2016. Reactive case-detection of malaria in Pailin Province, western Cambodia: lessons from a year-long evaluation in a pre-elimination setting. Malar J 15: 132.
25. Sturrock HJW, Novotny JM, Kunene S, Dlamini S, Zulu Z, Cohen JM, Hsiang MS, Greenhouse B, Gosling RD, 2013. Reactive case detection for malaria elimination: real-life experience from an ongoing program in Swaziland. PLoS One 8: e63830.

26. Steinhardt LC et al., 2017. Effectiveness of insecticide-treated bednets in malaria prevention in Haiti: a case-control study. Lancet Glob Health 5: e96-e103.

27. Kibret S, Alemu Y, Boelee E, Tekie H, Alemu D, Petros B, 2010. The impact of a small-scale irrigation scheme on malaria transmission in Ziway area, central Ethiopia. Trop Med Int Health 15: 41-50.

28. Coldiron ME, Von Seidlein L, Grais RF, 2017. Seasonal malaria chemoprevention: Successes and missed opportunities. Malar J 16: 481.

29. Guler JL, Rosenthal PJ, 2019. Mass drug administration to control and eliminate malaria in Africa: how do we best utilize the tools at hand? Clin Infect Dis 69: 287-289.

30. Hsiang MS et al., 2020. Active case finding for malaria: a 3-year national evaluation of optimal approaches to detect infections and hotspots through reactive case detection in the lowtransmission setting of Eswatini. Clin Infect Dis 70: 1316-1325.

31. Hsiang MS et al., 2020. Effectiveness of reactive focal mass drug administration and reactive focal vector control to reduce malaria transmission in the low malaria-endemic setting of Namibia: a cluster-randomised controlled, open-label, twoby-two factorial design trial. Lancet 395: 1361-1373.

32. Bennett A et al., 2020. A longitudinal cohort to monitor malaria infection incidence during mass drug administration in Southern Province, Zambia. Am J Trop Med Hyg 103: 54-65.

33. Stresman G, Whittaker C, Slater HC, Bousema T, Cook J, 2020. Quantifying Plasmodium falciparum infections clustering within households to inform household-based intervention strategies for malaria control programs: an observational study and meta-analysis from 41 malaria-endemic countries. PLoS Med 17: e1003370.

34. Elgoraish AG, Elzaki SEG, Ahmed RTE, Ahmed Al, Fadlalmula HA, Abdalgader Mohamed S, Abdallah NI, Abdelgadir O, Ageep TB, El-Sayed BB, 2019. Epidemiology and distribution of Plasmodium vivax malaria in Sudan. Trans $R$ Soc Trop Med Hyg 113: 517-524.

35. Deress T, Girma M, 2019. Plasmodium falciparum and Plasmodium vivax prevalence in Ethiopia: a systematic review and meta-analysis. Malar Res Treat 2019: 7065064. 